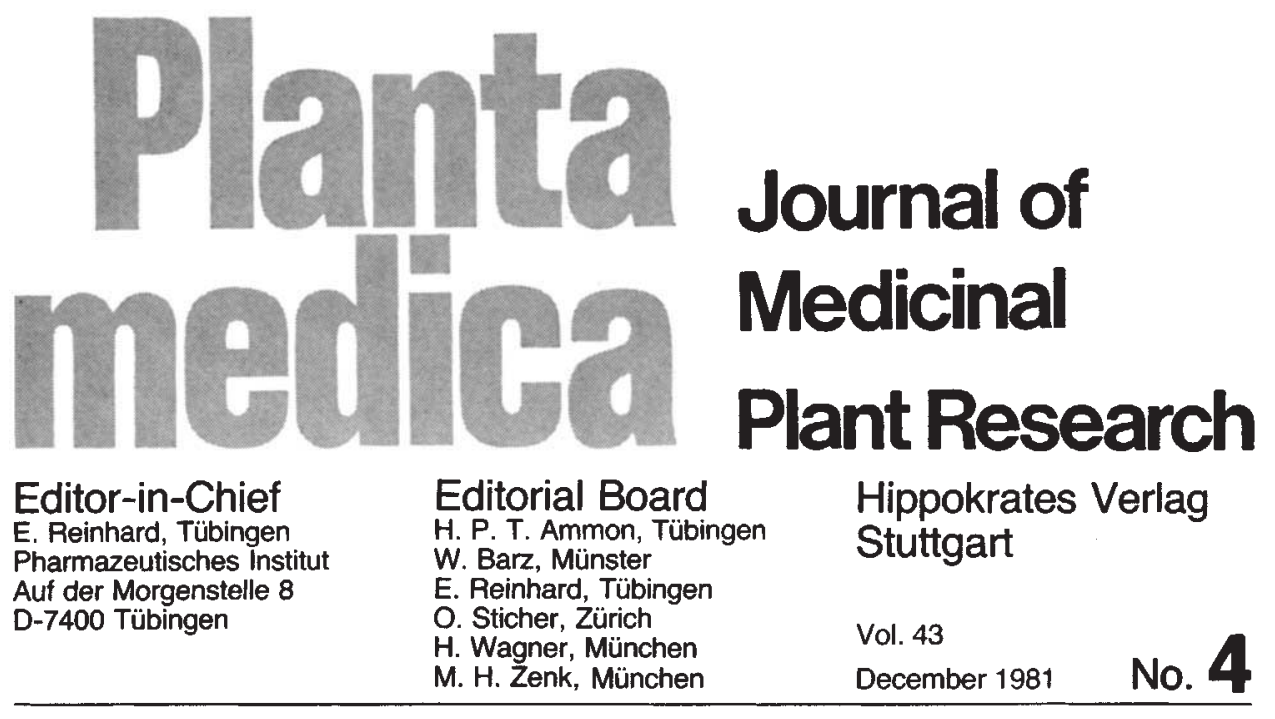

Rieview Anrtic|:

\title{
Crataegus, Toxikologie und Pharmakologie
}

Teil III: Pharmakodynamik und Pharmakokinetik

Crataegus, Toxicology and Pharmacology

Part III: Pharmacodynamics and Pharmacocinetics ${ }^{1}$

H. P. T. Ammon und M. Händel

Lehrstuhl Pharmakologie für Wissenschaftler, Fakultät für Chemie und Pharmazie der Universität Tübingen, Bundesrepublik Deutschland

\section{Stoffwechsel}

Atmung und Gesamtstoffwechsel

Bei Hunden blieben Atemvolumen und Sauerstoffverbrauch nach intravenöser Verabreichung von $2 \mathrm{ml}$ Esbericard $^{\circledast}$ praktisch unbeeinflußt (HokKERTS U. MÜLKE, 1955). Dem stehen Be-

1 Teil II: Pharmakodynamik, Planta Medica 43, 209 (1981). funde mit Heptaoxyflavanglykosid gegenüber, bei denen nach intravenöser Injektion von 19 bis $35 \mathrm{mg} / \mathrm{kg}$ beim Hund eine Steigerung des Atemvolumens von $21-82 \%$ beobachtet wurde (Hille u. Mitarb., 1955). Allerdings liegen diese Dosen teilweise 100 mal höher als die häufig am Herz-Kreislauf-System angewandten (HILle et al., 1955; ENGELKING U. WIILIG, 1958).

Bei der Maus führten $5 \mathrm{mg} / \mathrm{kg}$ i.p. von oligomeren Procyanidinen für etwa 
1 Stunde zu einer Temperatursenkung um $1-2^{\circ} \mathrm{C}$ (REWERSKI u. Mitarb., 1971).

\section{Lipidstoffwechsel}

Störungen im Lipidstoffwechsel, insbesondere Hypercholesterinämie und Hyperlipoproteinämie, gelten als Risikofaktoren bei der Entstehung degenerativer Gefäßerkrankungen, insbesondere auch der Coronarsklerose. Ziel der Therapie auf lange Sicht ist es, durch geeignete Maßnahmen einschließlich der Verabreichung von Pharmaka erhöhte Lipidspiegel zu senken. Der Einfluß von wäßrigen und alkoholischen Crataeguspräparationen auf die Konzentration der Blutlipide wurde bisher nicht untersucht. Nach oligomeren Procyanidinen niedrigen Polymerisationsgrades (10 $\mathrm{mg} / \mathrm{kg}$ i.v.) fand sich bei der Ratte nach 1 Stunde eine signifikante Senkung des Cholesterinspiegels und der Serumtriglyzeride (MAGDA, 1975).

Beim Kaninchen konnten solche Effekte allerdings nicht beobachtet werden (MAGDA, 1975).

Die Verabreichung von Cholesterin mit der Nahrung an Kaninchen dient als Modell zur Erzeugung einer experimentellen Hypercholesterinämie sowie arteriosklerotischer Veränderungen der Gefäße. Bei Kaninchen, die über längere Zeit auf diese Weise behandelt waren und Serumcholesterinwerte um $650 \mathrm{mg}$ $\%$ und mehr aufwiesen, führte nach $\mathrm{Ab}$ setzen der Cholesterinzufuhr die tägliche Gabe von $0,5 \mathrm{ml} / \mathrm{kg}$ alkoholischen Extrakts aus Crataegus (1:1) zu einer allmählichen Senkung des Serumcholesterins. Auch die Lipidablagerungen gingen zurück (Gusseinow, 1963). Eine Abnahme des Serumcholesterins um ca. $100 \mathrm{mg} \%$ konnte unter ähnlichen Be- dingungen auch bei täglicher Zufuhr von $30 \mathrm{mg} / \mathrm{kg}$ Crataegussaponinen mit dem Futter beobachtet werden (GusseINow, 1965). Bei Hühnern verhinderte die wöchentliche 2malige intravenöse Gabe von 1,5 ml Crataegutt ${ }^{\circledR}$ die durch Cholesterinfütterung hervorgerufenen arteriosklerotischen Veränderungen der Aorta sowie die Cholesterinansammlung in der Leber (Heinitz, 1963). Das Ergebnis ist durch Vergleich mit einer Kontrollgruppe, die normales Futter bzw, einer anderen Gruppe, die Cholesterin zugefüttert bekam, abgesichert. Diese Befunde lassen auf eine gewisse Schutzwirkung von peroral aufgenommenen Crataeguspräparationen gegenüber alimentärer Hypercholesterinämie im Tierversuch schließen.

\section{Körperliche Leistung}

Eine Methode, den Einfluß von Stoffen auf die körperliche Leistungsfähigkeit im Tierversuch zu prüfen, stellt der Schwimmversuch dar. Bei Meerschweinchen wurde die mittlere Schwimmdauer von ca. 37 Minuten durch Vorbehandlung mit Esbericard ${ }^{\oplus}$ (keine Angabe der Dosierung bzw. der Behandlungsdauer) nicht signifikant beeinflußt. Esbericard ${ }^{\circledR}$ blieb dabei auch ohne Einfluß auf die durch die Schwimmbelastung hervorgerufene Zunahme des Herz- und Nebennierengewichtes (WERNER, 1956). Zu ähnlichen Befunden kamen LüBBERS und Kuschke (1955) bei Ratten, die 13 Tage je $1 \mathrm{mal}$ pro Tag bis zur Erschöpfung geschwommen hatten, wenn diesen Tieren eine Woche vor und während der Versuche 3 Tropfen Crataegusextrakt oder $1 \mathrm{mg}$ Triterpensäure pro Tier peroral verabreicht wurden. Auch SEIDL und STARLINGER (1956) konnten nach 
peroraler Verabreichung von Crataegussäuren mit dem Futter keine Steigerung der Schwimmleistung beobachten. Eine signifikante Zunahme der Schwimmdauer wurde dagegen von BöHM (1956) nach mehrtägiger i.p.-Behandlung mit Flavonlösungen $(1 \%, 0,2$ $\mathrm{ml} / 100 \mathrm{~g}$ ) beobachtet.

\section{Zentralnervensystem}

Untersuchungen am zentralen Nervensystem wurden mit oligomeren Procyanidinen durchgeführt. Bei der Maus führten 2 bzw $5 \mathrm{mg} / \mathrm{kg}$ i.p. zu einer signifikanten Verlängerung der Hexobarbitalschlafzeit. Die gleichen Dosen bewirkten eine Senkung der Aggressivität (REWERsKi u. Mitarb., 1971). Dies weist auf eine zentral dämpfende Komponente dieser Substanzen hin.

\section{Glatte Muskulatur \\ Darm}

Am isolierten Meerschweinchendünndarm fand Kumakura (1967) eine Hemmung der kontraktionsauslösenden Wirkung von Histamin, Acetylcholin, Serotonin, Bradykinin und Nikotin durch Crataegutt $^{\otimes}(0,1 \mathrm{ml} / \mathrm{ml})$; die Spontanmotilität des Kaninchendünndarms wurde dagegen nicht beeinflußt. Untersuchungen mit Referenzsubstanzen fehlen.

\section{Uterus}

Eine Herabsetzung des Tonus des isolierten Uterus von Katze, Kaninchen und Meerschweinchen durch alkoholische Crataegustinktur wird von GRAham (1940) berichtet. Der gleiche Autor fand auch eine Hemmung der Motilität des schwangeren Uterus nach intravenöser Verabreichung von $0,1 \mathrm{ml} \mathrm{Cra-}$

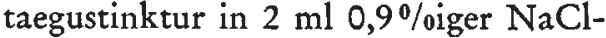
Lösung. Berücksichtigt man die coronardilatierende Wirkung von Crataeguspräparationen und die Steigerung der Muskeldurchblutung, so läßt sich ganz allgemein an eine gewisse relaxierende Wirkung auf die glatte Muskulatur durch Crataeguspräparationen denken.

\section{Sonstige Wirkungen}

Bei 2 Hunden wurde nach alkoholischem Crataegusextrakt eine Steigerung der Gallen- und Speichelsekretion beobachtet (Gusseinow und Juzbasinskaja, 1961).

\section{Interaktionen mit Glykosiden}

In einer Arbeit aus dem Jahre 1962 beschreiben TRUNZLER und SCHULER eine Zunahme der Kontraktionsamplitude beim isolierten Meerschweinchenherz nach Langendorff bei Zusatz von $0,15 \mathrm{ml} \mathrm{Crataegutt}{ }^{\circledR}$ pro Infusion (Infusionsdauer 4,5 Min.). Eine Zunahme der Kontraktionsamplitude findet sich auch bei einer Kombination von $3 \mu \mathrm{g}$ Digitoxin bzw. $15 \mu \mathrm{g}$ Digoxin bzw. 8,5 $\mu \mathrm{g}$ Strophanthin mit $0,15 \mathrm{ml}$ Crataegutt $^{\circledR}$. Zwar schreiben die Autoren, daß es hierbei zu einer Verstärkung der Effekte kommt, doch sind diese Aussagen in der Arbeit nicht nachprüfbar, da quantitative Angaben über die Wirkung der Herzglykoside als solche fehlen.

Aufgrund der Tatsache, daß beim Meerschweinchen die Toxizität von Herzglykosiden (Digitalis lanata Reinglykosid, Extrakt aus Convallaria majalis, Digitoxin, Strophanthin) - sie wurden infundiert - durch vorherige perorale Gabe von $1 \mathrm{ml}$ alkoholischem Crataegusextrakt gesteigert wurde (es wurden die tödliche Mengen der Herz- 
glykoside und daraus die jeweiligen $\mathrm{ME} / \mathrm{ml}$ Glykosidlösung bestimmt), schließt SEMm (1952) auf eine Verstärkung der Herzwirkung der Herzglykoside durch Crataegus. Dies ist jedoch ein sehr indirektes Verfahren, das nach den heutigen experimentellen Möglichkeiten nicht als beweiskräftig angesehen werden kann.

Am isolierten Froschherz konnte KuzNecova (1969) eine Steigerung der Kontraktionsamplitude durch einen alkoholischen Crataegusextrakt $(0,15 \times$ $10^{-3} \mathrm{~g} / \mathrm{ml}$ ) um $11 \%$ erzielen, mit Strophanthin $\left(0,2 \times 10^{-5}\right.$ und $\left.7 \times 10^{-6} \mathrm{~g} / \mathrm{ml}\right)$, Digitoxin $\left(0,1 \times 10^{-5}\right.$ und $\left.7 \times 10^{-6} \mathrm{~g} / \mathrm{ml}\right)$ sowie Convallatoxin $\left(0,04 \times 10^{-5} \mathrm{~g} / \mathrm{ml}\right)$ alleine war keine Wirkung auf die Kontraktionsamplitude zu sehen. Lediglich mit $7 \times 10^{-6} \mathrm{~g} / \mathrm{ml}$ Convallatoxin nahm sie um 15\% zu. Die kombinierte Anwendung brachte lediglich mit Strophanthin $\left(0,2 \times 10^{-5} \mathrm{~g} / \mathrm{ml}\right)$ Digitoxin $\left(0,1 \times 10^{-5} \mathrm{~g} / \mathrm{ml}\right)$ und Convallatoxin $\left(7 \times 10^{-6} \mathrm{~g} / \mathrm{ml}\right)$ eine Steigerung auf insgesamt $25 \%$, die auch statistisch gesichert ist. Beim ermüdeten Froschherzen führt Crataegusextrakt zu einem 75$\%$ igen Anstieg der Amplitude. Von den Glykosiden hatten jeweils die niedrigen Konzentrationen keinen Effekt, die Steigerungen durch die höheren Konzentrationen liegen zwischen +16 und $+30 \%$. Eine Wirkungsverstärkung gegenuiber der Crataeguswirkung allein ergibt sich hier nur durch die niedrigere Strophanthin- bzw. die höhere Convallatoxindosis. Beim Digitoxin war die Gesamtwirkung eher geringer als die alleinige Crataeguswirkung.

Während sich aus den zu beurteilenden Ergebnissen der beiden ersten Ar- beiten eine gegenseitige verstärkende Wirkung nicht ableiten läßt, ist eine solche bei der Arbeit von Kuznecova (1969) zumindest zum Teil signifikant nachweisbar. $\mathrm{Da}$ man davon ausgehen kann, daß die Herzglykoside über einen identischen Wirkungsmechanismus verfügen, bleibt allerdings unklar, warum der sich gegenseitig verstärkende Effekt beim Digitoxin nicht beim ermüdeten Herzen auftritt. Untersuchungen am Warmblütlerherzen unter Verwendung von echten Dosiswirkungsbeziehungen und Einbeziehung entsprechender Kontrollen wären hier sicher hilfreich.

\section{Pharmakokinetik}

Untersuchungen über Resorption, Verteilung und Metabolismus von $\mathrm{Zu}-$ bereitungen aus Crataegus sowie von dessen Inhaltsstoffen liegen bisher nicht vor. Sie sind für Crataeguszubereitungen als solche auch nicht möglich, $\mathrm{da}$ es sich dabei um Gemische aus Wirkstoffen und anderen bei der Extraktion anfallenden Stoffen handelt.

$\mathrm{Da}$ eine exakte Standardisierung auf den oder die Wirkstoffe bisher nicht möglich war, bleibt nur der Versuch einer annäherungsweisen Beurteilung mit Hilfe der pharmakodynamischen Eigenschaften. Dabei stellen sich folgende Fragen:

1. Sind die verschiedenen Effekte einer Crataeguszubereitung, die eine therapeutische Relevanz haben, mit etwa gleichen Dosen zu erzielen?

2. Sind sie nicht nur nach parenteraler, sondern auch nach peroraler Applikation zu beobachten?

3. Ist die Wirkungsdauer derart, daß sie von therapeutischem Interesse ist? 
Für die wirksamen Inhaltsstoffe liegen zusammenhängende Untersuchungen nicht vor.

Ad 1. Die Frage, ob alle beobachteten Effekte mit vergleichbaren Dosen zu erzielen sind, ist nach den vorliegenden Ergebnissen nur sehr vorsichtig $\mathrm{zu}$ beurteilen, da in den seltensten Fällen alle interessierenden Parameter gleichzeitig unter denselben Bedingungen gemessen wurden. Es kann daher nur versucht werden, soweit dies überhaupt möglich und zulässig ist, eine halbquantitative Gegenüberstellung $\mathrm{zu}$ geben. Wie die Tabelle XVI zeigt, finden sich beim narkotisierten Hund nach intravenöser Verabreichung beim Esbericard ${ }^{\circledR}$ bei vergleichbaren Dosen eine Zunahme der coronaren Durchblutung, eine Abnahme des Blutdruckes, eine Zunahme der Muskeldurchblutung, eine Abnahme der Herzfrequenz und eine Zunahme der Kontraktilität. Bei einem Wirkstoffkonzentrat aus Crataegus haben

\section{Table XVI}

Dosage of Crataegus preparations and constituents during measurement of cardiovascular parameters of the anaesthesized dog after IV application

\begin{tabular}{|c|c|c|c|c|c|}
\hline preparation & $\begin{array}{l}\text { increase of } \\
\text { coronary } \\
\text { blood flow }\end{array}$ & $\begin{array}{l}\text { decrease of } \\
\text { blood pressure }\end{array}$ & $\begin{array}{l}\text { increase of } \\
\text { blood supply } \\
\text { of muscle }\end{array}$ & $\begin{array}{l}\text { decrease of } \\
\text { heart rate }\end{array}$ & $\begin{array}{l}\text { increase of heart } \\
\text { contractility }\end{array}$ \\
\hline Esbericard ${ }^{\circledR}$ & $2-4 \mathrm{ml}$ & $1 \mathrm{ml}$ & $0.025-2 \mathrm{ml}$ & $2-4 \mathrm{ml}$ & $2-4 \mathrm{ml}$ \\
\hline Crataegutt $^{\circledR}$ & $0.3-1.0 \mathrm{ml} / \mathrm{kg}$ & $0.3-1.0 \mathrm{ml} / \mathrm{kg}$ & - & - & - \\
\hline $\begin{array}{l}\text { concentrated } \\
\text { constituents } \\
\text { of Crataegus }\end{array}$ & 1.2 and $5 \mathrm{ml}$ & $5 \mathrm{ml}$ & - & $2-5 \mathrm{ml}$ & $2 \mathrm{ml}$ \\
\hline $\begin{array}{l}\text { Heptaoxyflavan } \\
\text { glycoside }\end{array}$ & - & $1-20 \mathrm{mg} / \mathrm{kg}$ & - & - & $0.5-15.0 \mathrm{mg} / \mathrm{kg}$ \\
\hline
\end{tabular}

-: no data available

\section{Table XVII}

Dosage of Cratacgus preparations and constituents during measurement of cardiovascular parameters of the anaesthesized cat after IV application

\begin{tabular}{llcccc}
\hline preparation & $\begin{array}{l}\text { increase of } \\
\text { coronary } \\
\text { blood flow }\end{array}$ & $\begin{array}{l}\text { decrease of } \\
\text { blood pressure }\end{array}$ & $\begin{array}{l}\text { increase of } \\
\text { blood supply } \\
\text { of muscle }\end{array}$ & $\begin{array}{l}\text { decrease of } \\
\text { heart rate }\end{array}$ & $\begin{array}{l}\text { increase of heart } \\
\text { contractility }\end{array}$ \\
\hline $\begin{array}{l}\text { alcoholic extract } \\
\text { of Crataegus }\end{array}$ & - & $0.1-1.0 \mathrm{ml} / \mathrm{kg}$ & - & $0.5 \mathrm{ml} / \mathrm{kg}$ & $0.5 \mathrm{ml} / \mathrm{kg}$ \\
$\begin{array}{l}\text { total flavonoids } \\
\text { total saponins }\end{array}$ & $50-10 \mathrm{mg} / \mathrm{kg}$ & $10 \mathrm{mg} / \mathrm{kg}$ & - & - & $10 \mathrm{mg} / \mathrm{kg}$ \\
\hline
\end{tabular}

-: no data available 
wir ähnliche Verhältnisse. Hier wurde allerdings die Muskeldurchblutung nicht gemessen.

Bei der Katze (Tab. XVII) wurden bei einem alkoholischen Extrakt aus Crataegus sowie bei Gesamtflavonoiden 3 Parameter, nämlich Blutdruck, Herzfrequenz und Kontraktilität bzw. Coronardurchfluß, Blutdruck und Kontraktilität nach deren intravenöser Verabreichung gemessen. Wie die Tabelle XVII zeigt, wurden dabei Anderungen der erwähnten Parameter mit ähnlichen Dosen erzielt.

Ad 2. Zur Frage der peroralen Wirkung liegen uns die Arbeiten von $\mathrm{MÄ}-$ VERS und HENSEL (1974) sowie RodDEwig und Hensel (1977) vor. Sie fanden beim wachen Hund nach peroraler Gabe von Crataegutt ${ }^{\circledast}$ bzw. oligomeren Procyanidinen eine Zunahme des Coronardurchflusses. Uber den Einfluß auf Blutdruck (Ausnahme: geringe Senkung bei oligomeren Procyanidinen), periphere Durchblutung, Herzfrequenz und Kontraktilität des Herzmuskels sind jedoch keine Angaben zu finden. Aufgrund des vorliegenden tierexperimentellen $\mathrm{Ma}$ terials ist es denkbar, daß Crataeguspräparationen oder -inhaltsstoffe auch bei anderen Parametern Wirkung zeigen.

Aus Untersuchungen von LAPARRA et al. (1977) mit ${ }^{14} \mathrm{C}$-oligomeren Procyanadinen geht hervor, daß diese nach oraler Gabe resorbiert werden. Das Maximum der Radioaktivität im Blut bei der Maus wurde nach 45 Minuten gemessen. Die Halbwertszeit betrug 5 Stunden. Flavonoide werden nach BöHM (1959) enteral resorbiert.

Ad 3. Für die Wirkungsdauer ergibt sich folgendes Bild: a) intraarteriell verabreicht, findet sich bei Anwendung von Crataeguspräparationen und -inhaltsstoffen in vivo eine Wirkungsdauer von:

1-10 Minuten (Tab. VII) beim coronaren Durchfluß (intracoronar), 2-5 Minuten (Tab. VIII) beim Blutdruck,

1-3 Minuten (Tab. IX) bei der peripheren Durchblutung,

b) intravenös verabreicht, ist eine Wirkungsdauer angegeben von:

ca. 20 Minuten (Tab. VII) beim coronaren Durchfluß,

ca. 10 Minuten (Tab. VIII) beim Blutdruck,

ca. 10 Minuten (Tab. IX) bei der Muskeldurchblutung,

ca. 90 Minuten (Tab. XI) bei der Herzfrequenz.

c) Peroral verabreicht wurde von $\mathrm{M} \ddot{\mathrm{A}}$ VERS und HENSEL (1974) nach Gabe von $\mathrm{Crataegutt}^{\otimes}$ eine Steigerung des Coronardurchflusses über einen Zeitraum von ca. 1-2 Stunden beschrieben und bis ca. 4 Stunden (abhängig von der Dosis) fanden RODDEwIG und Hensel eine Steigerung des Coronardurchflusses nach peroraler $\mathrm{Zu}$ fuhr von oligomeren Procyanidinen (Tab. VII).

Während also die Effekte nach intraarterieller und intravenöser Gabe etwa gleich kurz sind und therapeutisch wenig Relevanz haben dürften, muß die relativ lange Wirkungsdauer nach peroraler Aufnahme (1-2 Stunden, abhängig von der Dosis) beim coronaren Durchfluß doch Aufmerksamkeit erregen und es wäre unbedingt erforderlich, entsprechende Untersuchungen auch bezüglich Herzfrequenz, Blutdruck, peripherer Durchblutung und Kontraktili- 
tät anzustellen. Die unterschiedliche Wirkungsdauer bei den verschiedenen Arten der Verabreichung ist einmal durch die etwa 2-3mal höheren Dosen bei peroraler Zufuhr zu erklären, zum zweiten könnte die Resorptionszeit (sie ist nicht bekannt) derart sein, daß sie eine längere Wirkungsdauer bedingt. Bei Berücksichtigung der Zeit-Wirkungskurven ist eine Resorptionszeit (abhängig von der Dosis) von 1/2-2 Stunden denkbar (Mävers und Hensel, 1974; Roddewig und Hensel, 1977).

Die kurze Wirkungsdauer bei intraarterieller und intravenöser Applikation spricht dafür, daß die wirksamen Inhaltsstoffe rasch eliminiert und/oder verteilt werden.

Es hat insgesamt gesehen den Anschein, als ob alle gewünschten Effekte auf Herz und Kreislauf bei den Extrakten aus Crataegus bei ähnlichen Dosen auftreten. Für die Inhaltsstoffe kann dies bisher nur bei den Gesamtflavonoiden angenommen werden.

\section{Summary}

\section{Toxicity}

The acute parenteral toxicity $\left(\mathrm{LD}_{50}\right)$ of Crataegus preparations and constituents was found to be in the range $18-34 \mathrm{ml} / \mathrm{kg}$ and $50-2600 \mathrm{mg} / \mathrm{kg}$ respectively; the oral acute toxicity was $18.5-33.8 \mathrm{ml} / \mathrm{kg}$ and $6 \mathrm{~g} / \mathrm{kg}$ respectively. No sufficient information is available concerning chronic toxicity, toxicity to foetus; generation studies and cancerogenesis.

\section{Pharmacodynamics}

1. Preparations of Crataegus are used in a) minor forms of coronary heart disease b) minor forms of heart failure

c) cardiac arrythmia.

2. From experiments with animals, preparations of Crataegus exhibited the following pharmacological effects which may be related to their therapeutical use (for scientific quality of experimental studies see tables).

a) for coronary heart disease:

- increase of coronary blood flow in vitro and in vivo (also observed after administration of 1-epicatechin, heptaoxyflavanglycoside, oligomeric procyanidins, hyperosides, total flavonoids. The effect of triterpenic acids is controversial.

- Decrease of arterial blood pressure (also observed with the use of heptaoxyflavanglycoside, oligomeric procyanidins and total saponins)

- increase of skeletal muscle blood flow (peripheral blood flow)

(also observed after heptaoxyflavanglycoside)

- decrease of heart rate in vivo; increase of heart rate in vitro

b) for heart failure:

little increase of contractility of heart muscle in vitro and in vivo (also observed using polymer flavans, oligomeric procyanidins, heptaoxyflavanglycoside).

\section{Pharmacokinetics}

No informations on blood levels of constituents of Crataegus are available.

1. Following parenteral administration of Crataegus extracts and polymers of flavans the above described pharmacodynamic effects appear at similar doses. Other constituents of Crataegus have not been tested for all of the above parameters. 
2. The duration of action is within 10 minutes following parenteral administration of the doses used in the available studies.

In the dog receiving a Crataegus preparation by oral route, coronary blood flow was increased over a period up to 2 hours.

Oligomeric procyanidins exhibit enteral absorption.

\section{Literaturverzeichnis}

1. Anguissola, A.: unveröffentlichte Daten (1970).

2. Assmann, E.: Crataegus oxyacantha. Deutsche Zeitschrift für Homöopathie 9, 248, 278, 301 (1930).

3. Bersin, T., A. Müller, H. Schwarz: Crataegus oxyacantha L. Arzneimittelforschung 5, 490 (1955).

4. Bezruk, P. I.: Pharmakologische Eigenschaften des Flacrasids. Pharmakol. i Toksikol. 30, 437 (1967).

5. Böhm, K.: Die Flavonoide. II. Resorption, Ausscheidung und Abbau der Flavonoide im Stoffwechsel. Arzneimittelforschung 9, 647 (1959).

6. Böhm, K.: Ergebnisse der Crataegus-Forschung. 2. Mitt.: Tierexperimentelle Befunde mit Gesamtauszügen und isolierten Reinsubstanizen aus Crataegus. Arzneimittelforschung 6, 38 (1956).

7. Braasch, W., W. Bienroth: Wirkung eines wäßrigen Crataegus-Extraktes auf den peripheren Kreislauf von Hunden. Arzneimittelforschung 10, 127 (1960).

8. Daweke, H., H. Giertz: Zur Pharmakologie von Crataegusextrakten (Esbericard( $\mathbb{B})$. Münchner Medizinische Wochenschrift 99, 1983 (1957).

9. Dörner, J., H. J. Kuschke: Besitzen die aus Crataegus gewonnenen Triterpensäuren (Crataegussäuren) eine pharmakodynamische Wirkung? Archiv für exper. Pathologie und Pharmakologie 225, 144 (1955).

10. Engelking, R., F. Willig: Die Wirkung eines Crataegus-Wirkstoffes (Heptahydroxy- flavanglykosid) in wäßriger Lösung auf Herz und Kreislauf von Hunden. Arzneimittelforschung 8, 39 (1958).

11. Graham, J. D. P.: Die Wirkungen von Crataegus oxyacantha. Quart. J. Pharmacy 13, 49 (1940).

12. Guendjev, Z.: Experimental Myocardial Infarction of the Rat and Stimulation of the Revascularization by the Flavonoid Drug Crataemon. Arzneimittelforschung 27 (8), 1576 (1977).

13. Gusseinow, D. J., P. A. Juzbasinskaja: Der Einfluß des flüssigen alkoholischen Extraktes von Crataegus pentagyna auf die Gallensekretion. Azerbajdzanskij medicinskij Zurnal, Baku 2, 60 (1961).

14. Gusseinow, D. J.: Die Wirkung eines Extraktes aus Crataegus pentagyna W. et $\mathrm{K}$. auf Cholesterin-induzierte Atherosklerose bei Kaninchen. Farmakol. i Toksikol. 26, 435 (1963).

15. Gusseinow, D. J.: Zur Pharmakologie der Gesamtsaponine von Crataegus pentagyna W. et K. Farmakol. i Toksikol. 28, 172 (1965).

16. Gusseinow, D. J.: Crataegus als Mittel gegen Arrhythmie. Kardiol, 6, 82 (1966).

17. Hahn, F., F. Klinkhammer, A. Oberdorf: Darstellung und pharmakologische Untersuchungen eines neuen therapeutischen Wirkstoffes aus Crataegus oxyacantha. Arzneimittelforschung 10, 825 (1960).

18. Heymanns, E., E. Frank: Klinische Kreislaufstudie über die Wirkung von Flavonen aus Crataegus bei Sauerstoff-Mangelbeatmung. Arztliche Forschung 10, 248 (1956).

19. Hille, H., H. Schaefer, H.-J. Teske, K. E. Zipf: Über Herz- und Kreislaufwirkung eines Crataegus-Wirkstoffes (Heptaoxyflavanglykosid). Archiv für exper. Pathologie und Pharmakologie 227, 281 (1955).

20. Hiyama, T., K. Tosaka: Akute Toxizität von Crataegus-Extrakten. Toho Igakkai Zasshi 16, 68 (1969).

21. Hockerts, T., G. Mülke: Beitrag zur Frage einer Coronarwirkung von wäßrigen Extrakten aus Crataegus-Droge. Arzneimittelforschung 5, 755 (1955).

22. Hutzel, H.: Kardiotherapeutikum Crataegus. Ärtliche Praxis 25, 3285 (1973). 
23. Jacobi, H., A. Oberdorf, W. Rummel: Zur Frage der Coronarwirkung von CrataegusExtrakten. Arzneimittelforschung 6, 98 (1956).

24. Kaczmarek, F., K. Mrugasiewicz, T. Wrocinski: Isolierung von Hyperosid und Vitexinrhamnosid aus Inflorescentia Crataegi und Untersuchungen über ihre Wirkung auf die Koronardurchblutung. Herba pol. 19, 138 (1973).

25. Kanno, T., T. Sugy, M. Yamamoto: Reduction of the Hypaxiainduced depression in the intracellular electrical activity of the ventricular muscle fibers of the rabbit fed on food containing Crataegutt ${ }^{\circledR}$. Jap. Heatt Journal 17, 512 (1976).

26. Kovách, A. G. B., M. Földi, L. Fedina: Die Wirkung eines Extraktes aus Crataegus oxyacantha auf die Durchströmung der Coronarien von Hunden. Arzneimittelforschung 9, 378 (1959).

27. Kukovetz, W.: Unveröffentlichte, wissenschaftliche Daten freundlicherweise von der Firma Schwabe, Karlsruhe, zur Verfügung gestellt (1978).

28. Kumakura, K., T. Oshima, H. Nishino: Pharmakologische Wirkungen des „Crataegutt". Unveröffentlichte Daten (1967).

29. Kuschke, H. J., H. Straub: Ober die Gefäßwirksamkeit wasserlöslicher Crataegussäuren. Archiv für exper. Pathologie und Pharmakologie 226, 547 (1955).

30. Kuznecova, L. V.: Kombinationswirkung von Herzglykosiden und Crataegus-Präparaten. Farmatsiya, Moskva 18, II/56 (1969).

31. Laparra, J., J. Michaud et J. Masquelier: Etude pharmacocinétique des oligomères flávanoliques. Plantes médicinales et phytothérapie 1977, Tome XI, n ${ }^{\circ}$ spécial, p. 133142.

32. Lübbers, F., H. J. Kuschke: Zum Problem des tierexperimentellen Nachweises einer Herzwirksamkeit vom Crataegus. Arzneimittelforschung 5, 603 (1955).

33. Mävers, W. H., H. Hensel: Veränderungen der lokalen Myokarddurchblutung nach oraler Gabe eines Crataegusextraktes bei nichtnarkotisierten Hunden. Arzneimittelforschung 24, 783 (1974).

34. Magda, S.: Prüfung von Crs-Blr 20441/0-3 am isolierten Meerschweinchen-Herzen nach
Langendorff. Unveröffentlichte, wissenschaftliche Daten freundlicherweise von der Firma Schwabe, Karlsruhe, zur Verfügung gestellt (1975).

35. Manolov, P., L. Daleva: Pharmakologische Untersuchungen eines Flavonoidgemisches aus Crataegus monogyna. Farmatsiya (Sofia) 19,38 (1969).

36. Manolov, P.: Pharmakologie von Crataemon. Suvrem. Med. 22, 20 (1971).

37. Nieschulz, O., K. Popendiker: Coronargefäß-erweiternde Wirkungen von Pflanzenextrakten. Arzneimittelforschung 7, 142 (1957).

38. Petrov, L., S. Gagov, A. Popova: Studies on the Vasoactive Effect of the Crataemon Preparation. Acta Physiologica et Pharmacologica Bulgarica 2, 82 (1974).

39. Pfannenstiel, W., F. Frief: Zur biologischen Standardisierung eines Crataegus-Präparates. Arztliche Forschung 12, I/570 (1958).

40. Rewerski, W., S. Lewak: Einige pharmakologische Eigenschaften der aus Weißdorn (Crataegus oxyacantha) isolierten FlavanPolymeren. Arzneimittelforschung 17, 490 (1967).

41. Roddewig, C., H. Hensel: Reaktion der 10kalen Myokarddurchblutung von wachen Hunden und narkotisierten Katzen auf orale und parenterale Applikation einer Crataegusfraktion (oligomere Procyanidine). Arzneimittelforschung 27, 1407 (1977).

42. Schimert, G.: Experimentelle Untersuchungen über die Wirkung von Crataegus oxyacantha auf die Durchblutung und die Dynamik des Herzens. Zeitschrift für die gesamte experimentelle Medizin 113, 113 (1943).

43. Schimert, G., H. Blömer: Beitrag zur Pharmakologie der Crataegus-Wirkstoffe. Uber die Kreislaufwirkung der T'riterpensäuren. Archiv für exper. Pathologie und Pharmakologie 217, 337 (1953).

44. Schwabe, W., R. Neu: Pharmakologische Prüfung einer aus Crataegus isolierten Substanz. Arzneimittelforschung 10, 60 (1960).

45. Seidl, E., H. Starlinger: Zur Frage der Wirkung von Crataegus auf Blutmilchsäure und Leistungsfähigkeit. Internat. Z. angew. Physiol. einschl. Arbeitsphysiol. 16, 303 (1956). 
46. Semm, K.: Die Wirkung von Crataegus für sich und ihre Verbindung mit Digitalis purpurea, Digitalis lanata, Adonis vernalis und Convallaria majalis auf das Herz des Meerschweinchens. Arzneimittelforschung 2, 562 (1952).

47. Stepka, W., A. D. Winters: A survey of the genus Crataegus for hypotensive activity. Lloydia 36, 436 (1973).

48. Thompson, E. B., G. H. Aynilian, P. Gora, N. R. Farnsworth: Preliminary Study of Potential Antiarrhytmic Effects of Crataegus monogyna. J. Pharmac. Sc. 63, 1936 (1974).

49. Trunzler, G., E. Schuler: Vergleichende Studien über Wirkungen eines Crataegus-Extraktes, von Digitoxin, Digoxin und g-Strophanthin am isolierten Warmblüterherzen. Arzneimittelforschung 12, 198 (1962).

50. Venturi: Exposé über die pharmakologischen Eigenschaften hinsichtlich der thera- peutischen Wirksamkeit und Verträglichkeit des Pflanzenextraktes, Cardiplant ${ }^{*}(\mathbb{B}$. Universita di Camerino, Instituto di Farmacologia, unveröffentlichte Daten (1963).

51. Vogel, G.: Vorhersehbarkeit der Wirkung von Drogenkombinationen - ja oder nein. Arzneimittelforschung 25, 1356 (1975).

52. Werner, H.: Klinisch-experimenteller Beitrag zur Frage der Wirksamkeit von Weißdornexrakten. Die Medizinische 18, 692 (1956).

53. Wezler, K.: Herzwirkung der CrataegusWirkstoffe. Arzneimittelforschung 8, 175 (1958).

Adresse: Prof. Dr. H. P. T. Ammon, Lehrstubl Pharmakologie für Naturwissenschaftler

Fakultät für Chemie und Pharmazie der Universität Tübingen: Auf der Morgenstelle 8, D-7400 Tübingen 1

\section{HOMOOPATHISCHE} FRISCHPFLANZEN

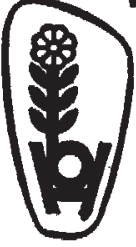

liefern wir seit über 40 Jahren aus biologischem Anbau

Für die Anbeuplanung 1981 erbitten wir baldige unverbindliche Bedarfameldung.

Bornträger + Schlemmer oHG 6521 OFFSTEIN 13

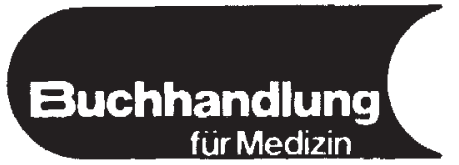

Postfach 593 - 7000 Stuttgart 1

liefert Ihnen jedes Buch aus dem medizinisch-naturwissenschaftlichen Berelch 